\title{
High-Range Angular Rate Sensor Based on Mechanical Frequency Modulation
}

\author{
Sergei A. Zotov, Member, IEEE, Alexander A. Trusov, Member, IEEE, Member, ASME, and \\ Andrei M. Shkel, Senior Member, IEEE
}

\begin{abstract}
We report, for the first time, an angular rate sensor based on mechanical frequency modulation (FM) of the input rotation rate. This approach tracks the resonant frequency split between two $X-Y$ symmetric high- $Q$ mechanical modes of vibration in a microelectromechanical systems Coriolis vibratory gyroscope to produce a frequency-based measurement of the input angular rate. The system is enabled by a combination of a MEMS vibratory high- $Q$ gyroscope and a new signal processing scheme which takes advantage of a previously ignored gyroscope dynamic effect. A real-time implementation of the quasi-digital angular rate sensor was realized using two digital phase-locked loops and experimentally verified using a silicon MEMS quadruple mass gyroscope (QMG). Structural characterization of a vacuumpackaged QMG showed $Q$ factors on the order of one million over a wide temperature range from $-40{ }^{\circ} \mathrm{C}$ to $+100{ }^{\circ} \mathrm{C}$ with a relative $x / y$ mismatch of $Q$ of $1 \%$. Temperature characterization of the FM rate sensor exhibited less than $0.2 \%$ variation of the angular rate response between $25^{\circ} \mathrm{C}$ and $70{ }^{\circ} \mathrm{C}$ environments, enabled by the self-calibrating differential frequency detection. High-speed rate table characterization of the FM angular rate sensor demonstrated a linear range of $18000 \mathrm{deg} / \mathrm{s}(50 \mathrm{r} / \mathrm{s}$, limited by the setup) with a dynamic range of $128 \mathrm{~dB}$. Interchangeable operation of the QMG transducer in conventional amplitudemodulated and new FM regimes provides a 156-dB dynamic range.

[2011-0161]
\end{abstract}

Index Terms-Frequency modulation (FM), gyroscope, rate sensor.

\section{INTRODUCTION}

$\mathbf{C}$ ONVENTIONAL vibratory rate gyroscopes are operated as analog amplitude modulation (AM) systems, where the mechanical sense-mode response is excited by the input angular rate that is amplitude modulated by the drive-mode velocity signal due to the Coriolis effect [1]. High mechanical quality factor $(Q)$ is critical for improving the sensitivity of micromachined gyroscopes [2], [3]. Mode matching of conventional high- $Q$ angular rate gyroscopes increases the signal-to-noise ratio at the tradeoff of linear input range and measurement bandwidth [4]. These constraints stem from a fundamental $Q$-versus-bandwidth tradeoff and dynamic range limitations of AM systems. The AM-based angular rate sensor operation

Manuscript received May 24, 2011; revised September 16, 2011; accepted November 8, 2011. Date of publication January 3, 2012; date of current version April 4, 2012. This work was supported by the Office of Naval Research (ONR)/Naval Surface Warfare Center Dahlgren Division (NSWCDD) under Grants N00014-09-1-0424 and N00014-11-1-0483. Subject Editor A. Seshia.

The authors are with the University of California, Irvine, CA 92697 USA (e-mail: szotov@uci.edu; atrusov@uci.edu; shkel@uci.edu).

Color versions of one or more of the figures in this paper are available online at http://ieeexplore.iee.org.

Digital Object Identifier 10.1109/JMEMS.2011.2178116

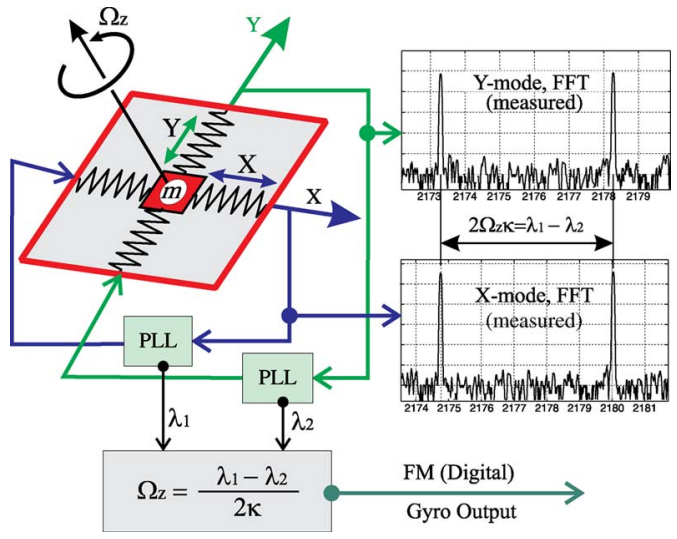

Fig. 1. Schematic of the closed-loop operated gyroscope based on the mechanical FM of the input angular rate. Inertial rotation causes a split between the gyroscope's $X$-mode and $Y$-mode, producing an FM measure of the input rate.

is also extremely sensitive to the value of the sense-mode $Q$ factor, resulting in significant scale factor drifts over practical variations in the ambient temperature and pressure [5]. In contrast, an angular rate sensor with intrinsic frequency modulation (FM) operation could eliminate the gain-bandwidth and dynamic range tradeoff of conventional AM gyroscopes and enable signal-to-noise ratio improvements by taking advantage of high- $Q$ mechanical sensor elements without limiting the measurement bandwidth [6]. In contrast to sensors with analog AM output, the frequency output of a quasi-digital FM sensor can be easily digitized without consuming A/D conversion resources [7]. At the same time, quasi-digital FM sensor architectures are known to provide inherent robustness against mechanical interferences and electromagnetic interferences (EMIs), since external perturbations (vibration, shock, and EMI) affect the signal amplitude only and do not change the frequency [8], [9].

Recently, we have reported a novel operating principle for a rate sensor which relies on tracking of the resonant frequencies of two high- $Q$ mechanical modes of vibration to produce a quasi-digital FM measurement of the input angular rate [10]. In this paper, we present a more complete analytical and experimental investigation, including range table characterization. This sensor architecture is enabled by a combination of a symmetric high- $Q$ silicon micromachined quadruple mass gyroscope (QMG) [11] and a new quasi-digital signal processing scheme [12], which takes advantage of a previously ignored mechanical FM effect of vibratory gyroscope dynamics (Fig. 1). 


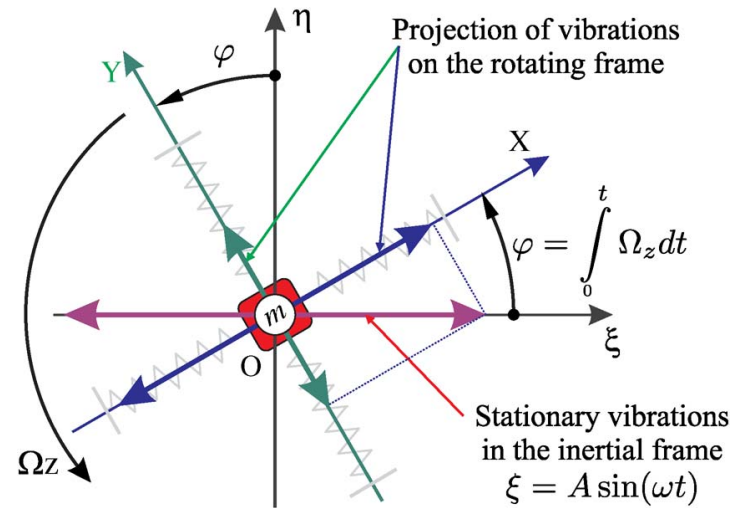

Fig. 2. Conceptual schematic of a $z$-axis gyroscope and relationship between the rotating and the inertial reference frames.

An explanation of the rate sensor based on mechanical FM is given in Section II, followed by a detailed description of the transducer design architecture in Section III. Section IV presents results of experimental demonstration of the mechanical FM phenomenon as well as rate characterization of the FM sensor. Section $\mathrm{V}$ concludes this paper with a discussion and a summary of the results.

\section{Frequency-Based Detection of Rate}

In this section, we analyze the often ignored effect of mechanical FM in vibratory gyroscopes [12], formulate a new FMbased principle of rate measurement, and analyze its properties.

\section{A. High-Q Gyroscope Dynamics}

Fig. 2 shows a conceptual schematic of a $z$-axis vibratory gyroscope considered as a proof mass $m$ suspended in the $X-Y$ plane of vibration and sensitive to the $z$-axis rate of rotation $\Omega_{z}$. Assuming negligible damping (i.e., high $Q$ ), the free vibration of a mode-matched device is governed by [13]

$$
\begin{aligned}
-\dot{\Omega}_{z} y(t)+\ddot{x}(t)+\left(\omega^{2}-\Omega_{z}^{2}\right) x(t)-2 \Omega_{z} \dot{y}(t) & =0 \\
\dot{\Omega}_{z} x(t)+\ddot{y}(t)+\left(\omega^{2}-\Omega_{z}^{2}\right) y(t)+2 \Omega_{z} \dot{x}(t) & =0
\end{aligned}
$$

where $\omega$ is the mechanical natural frequency for $\Omega_{z}=0$ input. In this analysis, we deliberately include the often ignored effect of the reverse Coriolis coupling of energy from the $Y$-mode back to the $X$-mode, as well as the centrifugal forces.

These governing equations (1) are written with respect to a rotating frame of reference $O X Y$ attached to the gyroscope die. In the inertial frame $O \xi \eta$, the gyroscope dynamics decouples into two separate equations

$$
\begin{aligned}
\ddot{\xi}(t)+\omega^{2} \xi(t) & =0 \\
\ddot{\eta}(t)+\omega^{2} \eta(t) & =0 .
\end{aligned}
$$

The mutual orientations of the inertial $O \xi \eta$ and rotating $O X Y$ frames of reference are shown in Fig. 2. The solution of (2) is

$$
\begin{aligned}
& \xi(t)=c_{1} \sin (\omega t)+c_{2} \cos (\omega t) \\
& \eta(t)=c_{3} \sin (\omega t)+c_{4} \cos (\omega t)
\end{aligned}
$$

where $c_{i}$ represents constants.

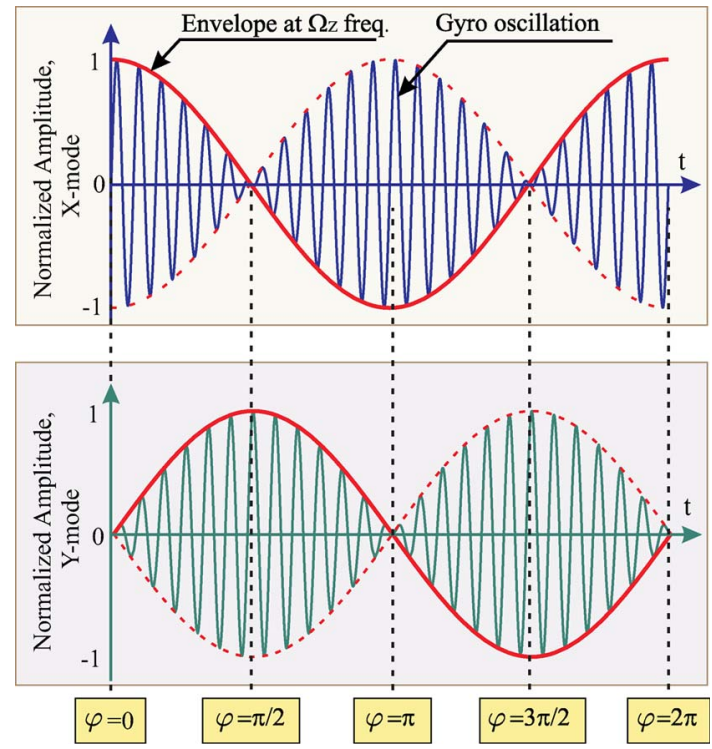

Fig. 3. Free vibration of an ideal mode-matched gyroscope with respect to the rotating frame of reference $O X Y$ showing two frequencies: The gyroscope's frequency $\omega$ and the rotation frequency $\Omega_{z}$.

Depending on the initial conditions, the solution of (3) corresponds to a pattern in the form of either a line or an ellipse. This pattern remains stationary in the inertial space. This phenomenon can be used for the realization of an angle gyroscope [14], [15] or an FM rate gyroscope [10].

The free vibration pattern (3) can be now mapped back to the rotating reference frame $O X Y$ attached to the gyroscope die

$$
\begin{aligned}
& x(t)=\xi(t) \cos \left(\int_{0}^{t} \Omega_{z} d t\right)+\eta(t) \sin \left(\int_{0}^{t} \Omega_{z} d t\right) \\
& y(t)=-\xi(t) \sin \left(\int_{0}^{t} \Omega_{z} d t\right)+\eta(t) \cos \left(\int_{0}^{t} \Omega_{z} d t\right) .
\end{aligned}
$$

A graphical plot of solution (4) for the case of a constant $\Omega_{z}$ is shown in Fig. 3. Using trigonometric identities, (4) is equivalent to

$$
\begin{aligned}
x(t)= & C_{1} \sin \left(\omega t+\int_{0}^{t} \Omega_{z} d t\right)+C_{2} \sin \left(\omega t-\int_{0}^{t} \Omega_{z} d t\right) \\
& +C_{3} \cos \left(\omega t+\int_{0}^{t} \Omega_{z} d t\right)+C_{4} \cos \left(\omega t-\int_{0}^{t} \Omega_{z} d t\right) \\
y(t)= & C_{1} \sin \left(\omega t+\int_{0}^{t} \Omega_{z} d t\right)-C_{2} \sin \left(\omega t-\int_{0}^{t} \Omega_{z} d t\right) \\
& -C_{3} \cos \left(\omega t+\int_{0}^{t} \Omega_{z} d t\right)+C_{4} \cos \left(\omega t-\int_{0}^{t} \Omega_{z} d t\right)
\end{aligned}
$$

where $C_{i}$ represents constants.

The solution (5) defines the gyroscope vibration as a superposition of two sinusoids with phases $\left(\omega t+\int_{0}^{t} \Omega_{z} d t\right)$ and 


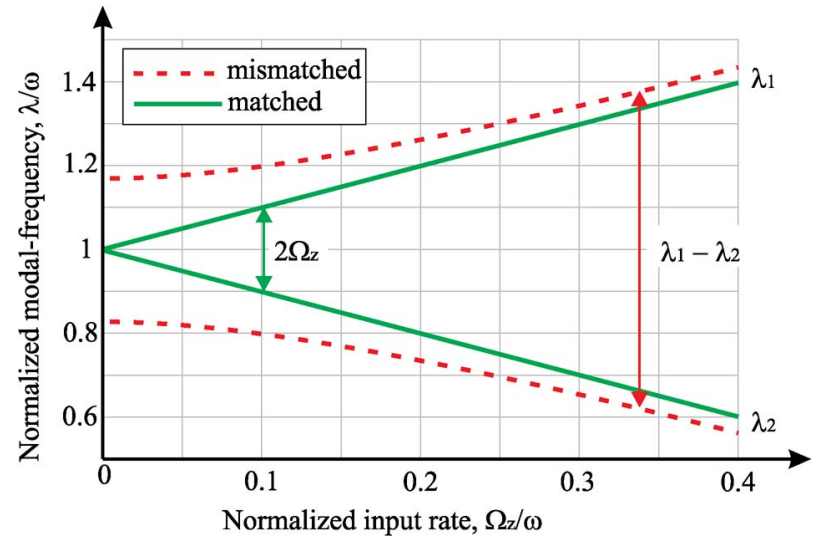

Fig. 4. FM of the gyroscope's modal frequencies $\lambda_{i}\left(\Omega_{z}\right)$ by the input angular rate $\Omega_{z}$ (simulation). An initially mode-matched gyroscope experiences a modal frequency split proportional to the rotation rate.

$\left(\omega t-\int_{0}^{t} \Omega_{z} d t\right)$, respectively. The effective modal frequencies $\lambda_{1}$ and $\lambda_{2}$ are defined by the time derivative of the phases with respect to time

$$
\begin{aligned}
& \lambda_{1}=\omega+\Omega_{z} \\
& \lambda_{2}=\omega-\Omega_{z} .
\end{aligned}
$$

The expression (6) defines the instantaneous modal frequencies $\lambda_{1}$ and $\lambda_{2}$ with respect to rotating reference frame $O X Y$ in the presence of arbitrary time-variant input $\Omega_{z} \neq 0$ (Fig. 4).

The gyroscope's free vibrations contain only one frequency $\omega$ with respect to the inertial frame (3). At the same time, two splitting frequencies $\lambda_{1}$ and $\lambda_{2}$ are observed (5) with respect to the device moving frame $O X Y$ (Fig. 3). The FM effect is inherent to the moving reference frame and enables measuring the input rate $\Omega_{z}$ from the observed split in the instantaneous modal frequencies.

The FM-based approach is also valid for nonideal gyroscopes with initial mismatch $\Delta \omega$ between two modes [10]. In this case, (6) transforms to

$$
\begin{aligned}
& \lambda_{1}=\sqrt{\Omega_{z}^{2}+\omega^{2}+\frac{\Delta \omega^{2}}{4}+\sqrt{\Delta \omega^{2}\left(\Omega_{z}^{2}+\omega^{2}\right)+4 \Omega_{z}^{2} \omega^{2}}} \\
& \lambda_{2}=\sqrt{\Omega_{z}^{2}+\omega^{2}+\frac{\Delta \omega^{2}}{4}-\sqrt{\Delta \omega^{2}\left(\Omega_{z}^{2}+\omega^{2}\right)+4 \Omega_{z}^{2} \omega^{2}}} .
\end{aligned}
$$

For the case of an initially mode-matched gyroscope (i.e., $\Delta \omega=0$ for $\Omega_{z}=0$ ), the solution (7) simplifies to the previously obtained (6).

These expressions for the instantaneous modal frequencies in the presence of time-variant rotation reveal that $\lambda_{1}$ and $\lambda_{2}$ of the gyroscope are modulated by the input angular rate $\Omega_{z}$ (Fig. 4). This rate-dependent change in modal frequencies presents a challenge for conventional high- $Q$ mode-matched gyroscopes because rotation results in mode detuning and loss of linearity.

The closed-form analytical solution (6) demonstrates that the instantaneous modal frequencies $\lambda_{1}$ and $\lambda_{2}$ are functions of only the instantaneous input angular rate $\Omega_{z}$. In contrast, the input angular acceleration $\dot{\Omega}_{z}$ does not affect the modal frequencies. A graphical demonstration of the gyroscope behavior for a time-varying angular rate $\Omega_{z}=\Omega_{0} \sin (\epsilon t)$, where

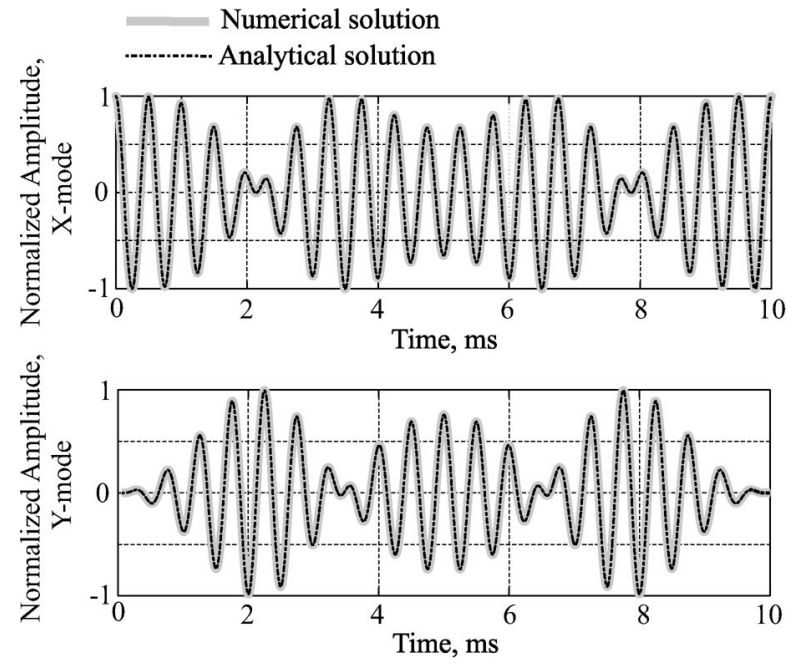

Fig. 5. Free vibration of an ideal mode-matched gyroscope with respect to the rotating frame of reference $O X Y$ for the case of a sinusoidal input rate with amplitude of $72000 \mathrm{deg} / \mathrm{s}(200 \mathrm{~Hz})$ and frequency of $100 \mathrm{~Hz}$.

$\Omega_{0}=200 \mathrm{~Hz}$ and $\epsilon=100 \mathrm{~Hz}$, is shown in Fig. 5. The solid line in Fig. 5 corresponds to the direct numerical solution of (1) for $\omega$ of $2 \mathrm{kHz}$ and the following initial conditions: $x(0)=1$, $\dot{x}(0)=0, y(0)=0$, and $\dot{y}(0)=0$. The dashed line represents the analytical solution in the form of a sum of two sinusoids with modal frequencies $\lambda_{1}=\omega+\Omega_{z}$ and $\lambda_{2}=\omega-\Omega_{z}$, obtained for the same parameters. The data show a complete overlap of the analytical and numerical solutions, verifying our analytical derivations for time-varying angular rates.

\section{B. Frequency-Based Detection of Input Rate}

In this section, we propose to take advantage of the modal frequency splitting to enable direct FM measurement of the input rotation rate. Based on the eigenfrequency solution (6) for an initially mode-matched gyroscope, the input rotation rate $\Omega_{z}$ can be measured from the observed modal frequencies $\lambda_{1}$ and $\lambda_{2}$ according to a linear expression

$$
\Omega_{z}=\frac{1}{2}\left(\lambda_{1}-\lambda_{2}\right) \quad \text { for } \Delta \omega=0 .
$$

The proposed FM-based operational principle can also be extended to the more general case of a gyroscope with an initial frequency mismatch $(\Delta \omega>0)$ by algebraically solving (7) for the input angular rate

$$
\Omega_{z}=\frac{1}{2} \sqrt{\left(\lambda_{1}-\lambda_{2}\right)^{2}-\frac{4 \omega^{2} \Delta \omega^{2}}{4 \omega^{2}+\Delta \omega^{2}-\left(\lambda_{1}-\lambda_{2}\right)^{2}}} .
$$

The obtained closed-form solutions (8) and (9) provide the basis for the frequency-based measurement of the input rate by tracking the modal frequency split in a high- $Q$ nearly modematched vibratory gyroscope. The quasi-digital FM approach resolves the $Q$ versus linear range and bandwidth tradeoff of conventional analog AM-based vibratory rate sensors. Maximization of the FM-operated gyroscope $Q$ factor improves the frequency stability and rate resolution. At the same time, 
the linear input range of an FM-operated gyroscope is independent of the $Q$ and is only limited by the device nominal frequency $\left(\Omega_{z}<\omega\right)$. The modal frequency split provides an instantaneous measure of the input rate, allowing the use of high- $Q$ structures without the sensor bandwidth sacrifice. An additional advantage of the approach is the self-calibration against common drifts in the modal frequencies enabled by the differential frequency measurement (i.e., $\lambda_{1}-\lambda_{2}$ ).

\section{High-Q Transducer Design}

In this section, we describe a symmetric high- $Q$ vibratory gyroscope structure tailored for FM operation.

\section{A. Design Requirements for FM Instrumentation}

Several design criteria must be met by the mechanical sensor element to fully realize the advantages of the proposed FM operation. Since the rate measurement relies on modal frequency tracking, a geometrically symmetric and mode-matched structure is needed to optimize the minimal detectable angular rate signal and increase the temperature stability of the sensor. High $Q$ factors allow achieving high frequency stability and low phase noise [16]. Identical high $Q$ is needed in both modes of mechanical vibration to maximize the modal frequency stability and rate resolution. These requirements are satisfied by an $X-Y$ symmetric dynamically balanced antiphaseoperated gyroscope, such as the recently introduced QMG architecture [11].

\section{B. $Q M G$ Architecture}

The mechanical structure of the QMG mechanical sensor element comprises four identical symmetrically decoupled tines with linear coupling flexures as well as a pair of antiphase synchronization lever mechanisms for both the $X$ - and the $Y$-modes (Fig. 6). This $X-Y$ symmetric system of four antiphase tines provides the structure with two antiphase dynamically balanced modes of mechanical vibration at a single operational frequency (Fig. 7). The complete $X-Y$ structural symmetry of the device improves robustness of frequency matching against the fabrication imperfections and temperature-induced frequency drifts. An additional advantage of the QMG antiphase levered architecture is the mechanical suppression of the parasitic common-mode inphase displacement in the coupled tines.

\section{EXPERIMENTAL CHARACTERIZATION}

In this section, we experimentally evaluate the FM-based rate detection approach using a vacuum-sealed QMG and a custom signal processing scheme.

\section{A. Prototype Fabrication and Packaging}

The QMG sensor element used for the experimental characterization was fabricated using an in-house single-mask waferscale silicon-on-insulator (SOI) process with a conductive

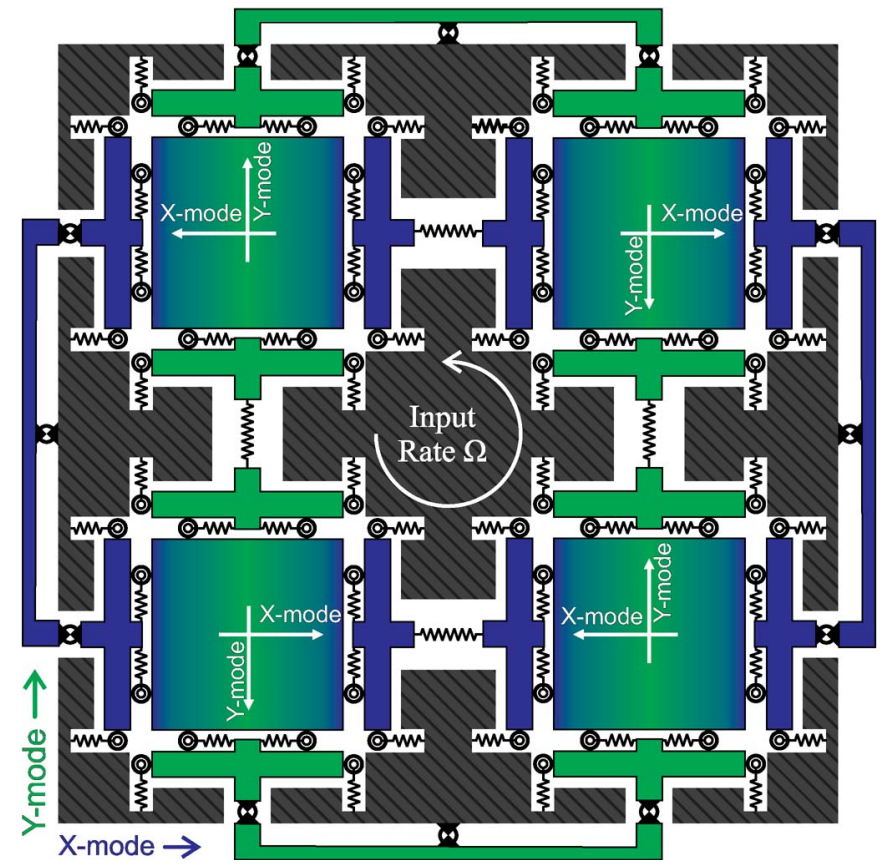

Fig. 6. Structural design of the symmetric dynamically balanced QMG mechanical transducer.

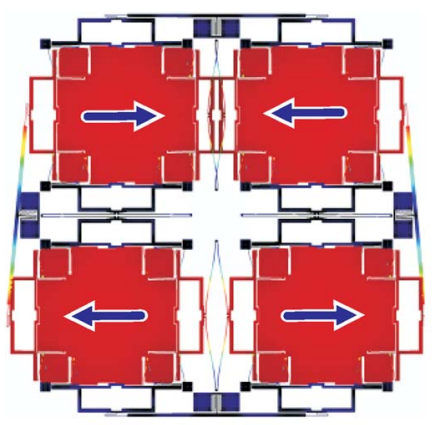

(a)

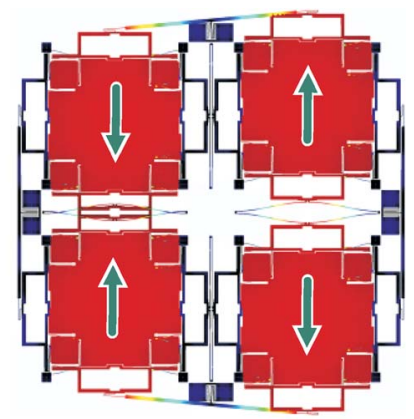

(b)
Fig. 7. QMG architecture provides balanced low-dissipation modes of $X$ - and $Y$-axis vibrations at the same frequency (FEM). (a) $X$-mode displacement. (b) $Y$-mode displacement

$100-\mu \mathrm{m}$-thick device layer and a $5-\mu \mathrm{m}$-thick buried oxide. First, a hard mask for sensor structures was defined by patterning a thermally grown $1-\mu \mathrm{m}$ layer of surface oxide with a Surface Technology Systems Advanced Oxide Etching tool. The gyroscope structures were then defined by deep reactive-ion etching using a Unaxis Versaline VL-7339 tool. The singulated sensors were released using a timed $20 \%$ hydrofluoric acid wet etch. The released QMG devices were bonded to ceramic dual in-line packages using $\mathrm{Au}-\mathrm{Sn}$ eutectic solder and wire bonded. To enable stand-alone high- $Q$ operation, the packaged sensors were vacuum sealed using custom-made glass lids with getter material [17] providing robust submillitorr vacuum inside the package cavities (Fig. 8).

\section{B. Structural Characterization}

The dynamically balanced antiphase design and vacuum packaging of QMG sensors were expected to yield isotropic high $Q$ factors for stable FM operation. The structural $X$ - and 


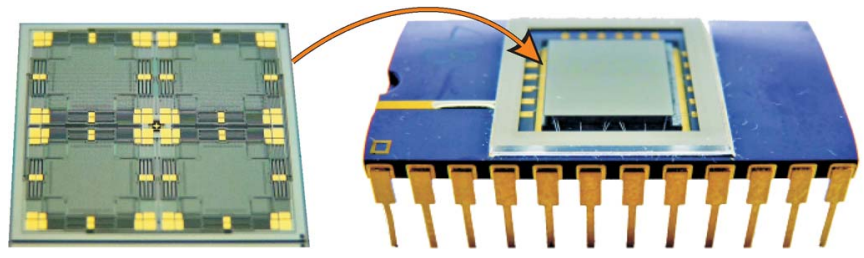

Fig. 8. Photograph of a SOI prototype of high- $Q$ QMG that is vacuum packaged at submillitorr cavity pressure using a custom lid with getters.

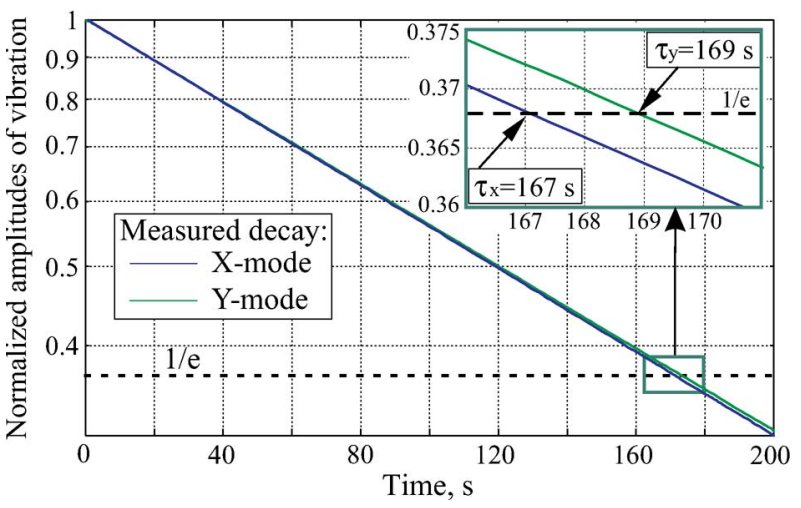

Fig. 9. Experimental characterization of the packaged QMG using ring-down test, revealing identical $Q$ factors for both modes (with $\Delta Q / Q$ of $1 \%$ ).

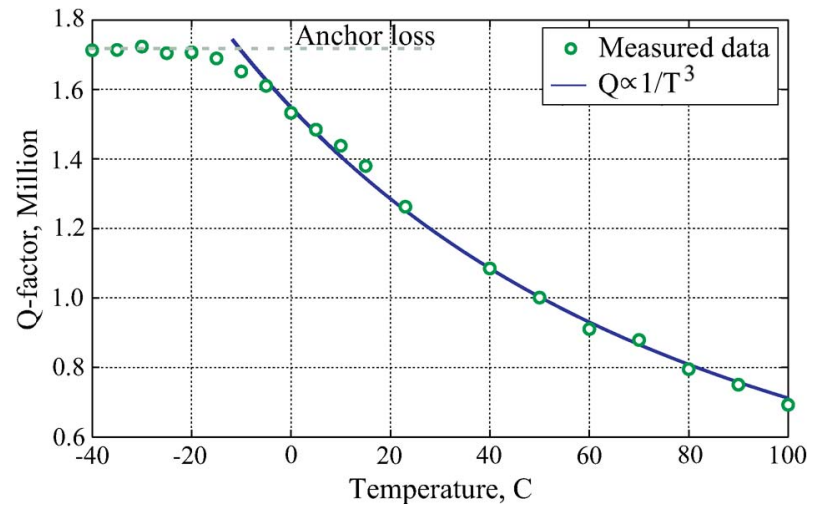

Fig. 10. Measured $Q$ factor versus temperature for $\mathrm{QMG}$, showing $1 / T^{3}$ dependence of thermoelastic dissipation for $T>0{ }^{\circ} \mathrm{C}$.

$Y$-modes of a vacuum-sealed QMG prototype were experimentally characterized at different temperatures using ring-down tests in a TestEquity 107 thermal chamber. Exponential fits of the time-domain amplitude decay data showed time constants of $\tau_{x}=167.1 \mathrm{~s}$ and $\tau_{y}=168.8 \mathrm{~s}$ for the $X$ - and $Y$-modes, respectively, confirming structural and damping symmetry, with $\Delta(1 / \tau)=6 \times 10^{-5} \mathrm{~s}^{-1}$ (Fig. 9). This value of time constant allows to observe free gyroscope dynamics during several minutes. The $Q$ factors were calculated by the formula $Q=$ $\pi f_{n} \tau$, with the measured natural frequency $f_{n}=2.2 \mathrm{kHz}$. A $Q$ factor of $X$ - and $Y$-modes of 1.16 million approaches the fundamental thermoelastic limit of 1.3 million obtained using finite-element modeling (FEM). Characterization of $Q$ factors over a $-40{ }^{\circ} \mathrm{C}$ to $+100{ }^{\circ} \mathrm{C}$ temperature range is shown in Fig. 10. For $T>0{ }^{\circ} \mathrm{C}$, the data exhibit $1 / T^{3}$ dependence, which is characteristic of thermoelastic dissipation [18]. Be-

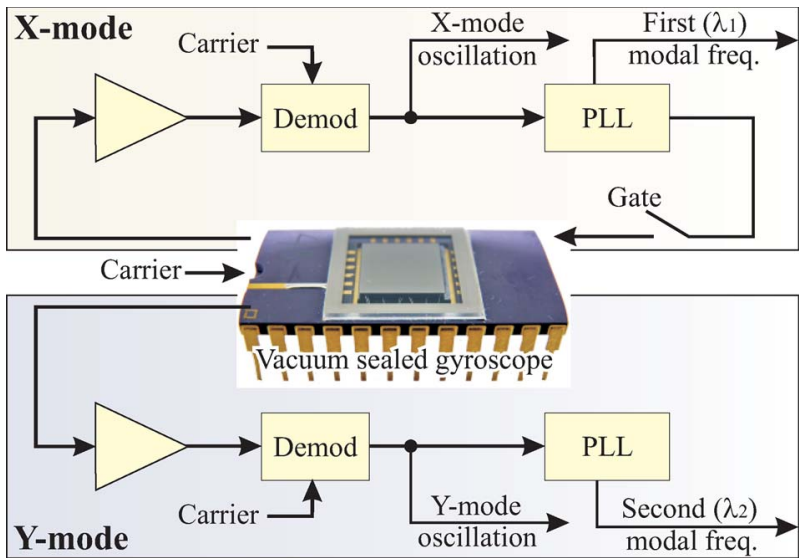

Fig. 11. Block diagram of QMG signal processing for mechanical FM measurements, showing $X$ - and $Y$-mode control loops.

low $0{ }^{\circ} \mathrm{C}$, the device $Q$ factor levels off at approximately 1.7 million, attributed to anchor loss caused by slight structural imbalances. A more detailed analysis of anchor loss due to structural imbalances in tuning-fork MEMS is reported in [19].

\section{Interface Electronic and Signal Processing}

For rate table characterization, a vacuum-packaged QMG is mounted to a printed circuit board containing signal detection electronics. Front-end amplification of the output signals is done using two transimpedance and instrumentation amplifiers for each $X$-mode and $Y$-mode. All postprocessing was implemented in real-time-DSP field-programmable gate array lock-in amplifier HF2 from Zurich Instruments. Separation of the useful signal from the feedthrough signal is accomplished using electromechanical AM (EAM), where a carrier voltage of $0.5 \mathrm{~V}$ at $52 \mathrm{kHz}$ is applied to the proof mass resulting in the AM of the motional signal [20]. The modulated signals of both the $X$ - and $Y$-modes were fed to phase-locked loops (PLLs) with the following parameters: a proportional gain of $0.3 \mathrm{~Hz} / \mathrm{deg}$, a time constant of $50 \mathrm{~ms}$, a central frequency of $2177 \mathrm{~Hz}$ (equal to the mechanical frequency $\omega$ ), and a range of $50 \mathrm{~Hz}$. These parameters provided a PLL bandwidth of $10 \mathrm{~Hz}$. These are used to track the natural frequencies and to provide resonant closed-loop excitation (Fig. 11). Gated excitation is used to open and close the excitation loop for each $X$-mode. When the gate is closed, the excitation is applied, and during this phase, no measurement is performed. When the gate is open, the signals are monitored to detect the difference in frequencies due to an input rotation rate. A practical FM system can be implemented using either free vibration of the proof mass or closed-loop excitation to maintain the vibrations. While both methods were implemented and experimentally verified, the focus of this paper is on the demonstration of the fundamental principle of operation using free vibrations.

\section{Demonstration of FM Phenomenon}

Section II theoretically investigated the typical modal frequency splitting in a vibrating gyroscope and its application to rate sensing. In order to experimentally demonstrate the 


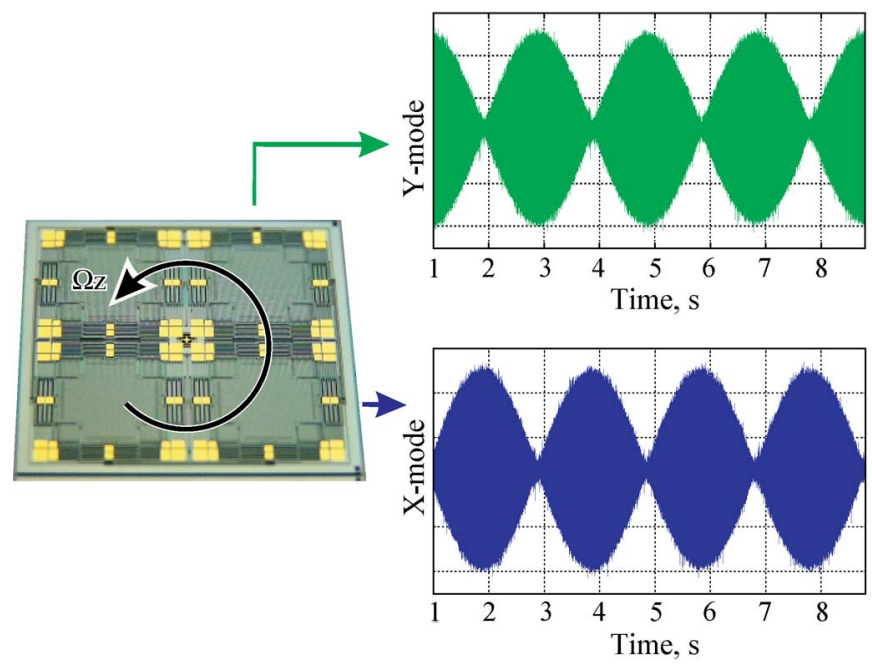

Fig. 12. Measured output of $X$ - and $Y$-modes of a vacuum-sealed QMG experimentally illustrates the frequency split phenomenon.

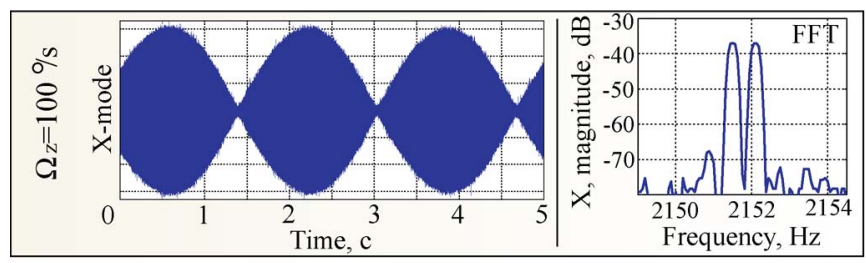

(a)

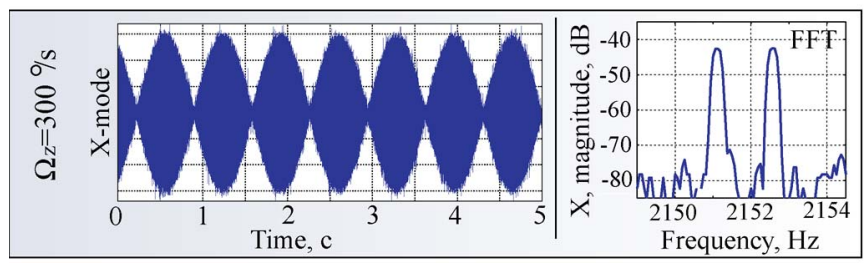

(b)

Fig. 13. Measured output of $X$-mode of a vacuum-sealed QMG in time and frequency domains for two different input rates. (a) $\Omega_{z}$ of $100 \mathrm{deg} / \mathrm{s}$ causes $0.48-\mathrm{Hz}$ frequency split. (b) $\Omega_{z}$ of $300 \mathrm{deg} / \mathrm{s}$ causes $1.44-\mathrm{Hz}$ frequency split.

effect of mechanical FM on the input rotation rate, a vacuumpackaged gyroscope with a mismatch of about $0.1 \mathrm{~Hz}$ was tested on an Ideal Aerosmith 2102 rate table. Initially, the rate table is stationary, and one of the gyroscope's modes ( $X$-mode) is excited into resonance by closing the gate (Fig. 11). The gate is then opened to allow free vibration of the gyroscope, and an input angular rate was applied. Acquisition of the $X$ and $Y$-modes of free vibration is done using a LeCroy WaveRunner 64Xi oscilloscope, while simultaneously conducting a fast Fourier transform (FFT) analysis with an HP 35665A dynamic signal analyzer. Fig. 12 shows the vibration in the form of beating for both the $X$ - and $Y$-modes as the gyroscope is rotated at $\Omega_{z}=100 \mathrm{deg} / \mathrm{s}$. Fig. 13 shows the $X$-mode output signal in both the time and frequency domains during rotations with 100- and 300-deg/s angular rates. From the FFTs shown in the right part of Fig. 13, it is evident that the split in frequencies is proportional to the rate of rotation. The measured data in Figs. 12 and 13 are an experimental demonstration of the ratedependent modal frequency splitting described in Section II.

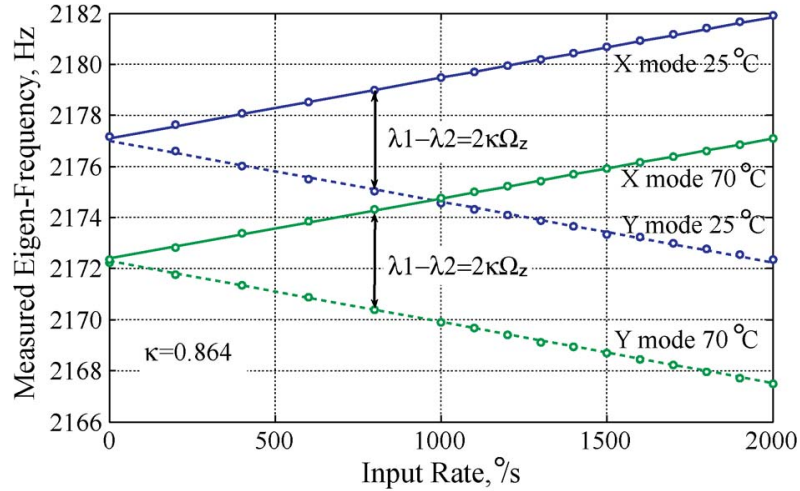

(a)

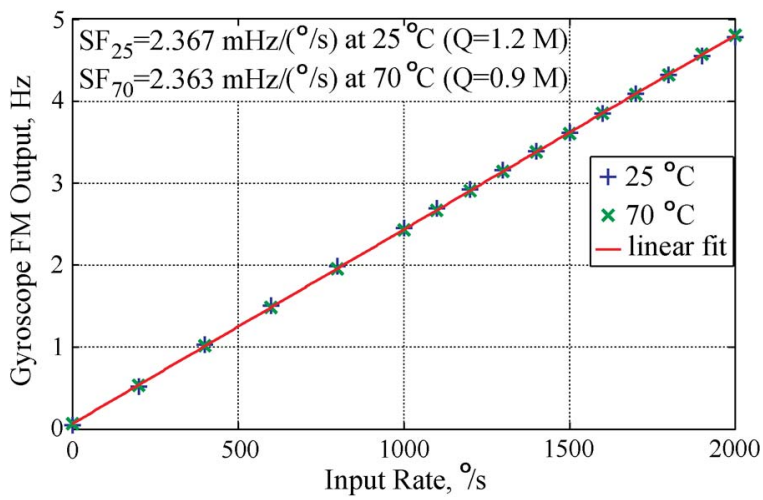

(b)

Fig. 14. Characterization of the FM-based sensor reveals no response drift between $25{ }^{\circ} \mathrm{C}$ and $70{ }^{\circ} \mathrm{C}$ (without any temperature compensation) despite a $30 \% Q$ reduction and a $5-\mathrm{Hz}$ drop of nominal frequency. (a) Differential FM detection of the $\Omega_{z}$ input rate from the modal frequency split $\left(\lambda_{1}-\lambda_{2}\right)$ is invariant to temperature. (b) Measured FM rate responses for $25^{\circ} \mathrm{C}$ and $70{ }^{\circ} \mathrm{C}$ using differential detection of the modal frequency split with inherent self-calibration.

\section{E. Rate Characterization}

In conventional AM-operated vibratory rate gyroscopes, drifts of modal frequencies and $Q$ factors are major sources of sensor output scale factor and bias drift over temperature. Theoretical analysis of the proposed FM rate sensor suggests immunity against these drift mechanisms by virtue of the differential frequency detection, i.e., measuring the frequency split by (8) or (9). To experimentally investigate this hypothesis, a vacuum-packaged QMG sensor instrumented for real-time closed-loop FM operation was characterized on a temperaturecontrolled Ideal Aerosmith 1291BR rate table at $25{ }^{\circ} \mathrm{C}$ and at $70{ }^{\circ} \mathrm{C}$. For these experiments, the $X$ - and $Y$-modes of the QMG device were electrostatically excited into resonances using a combination of $0.1-\mathrm{Vdc}$ bias and 0.1-Vac driving signals produced by two separate digital PLLs. The motional signals for both modes of vibration were detected using capacitive detection with EAM. The two modal frequencies of the gyroscope mechanical structure were continuously monitored by the two PLLs (Fig. 11). As theoretically expected for a modematched gyroscope, the measured split between the nominally equal modal frequencies was directly proportional to the input rate [Fig. 14(a)]. Unlike the conventional AM approach, the FM detection of the input rate from the modal frequency split demonstrated invariance to changes in temperature and $Q$ 


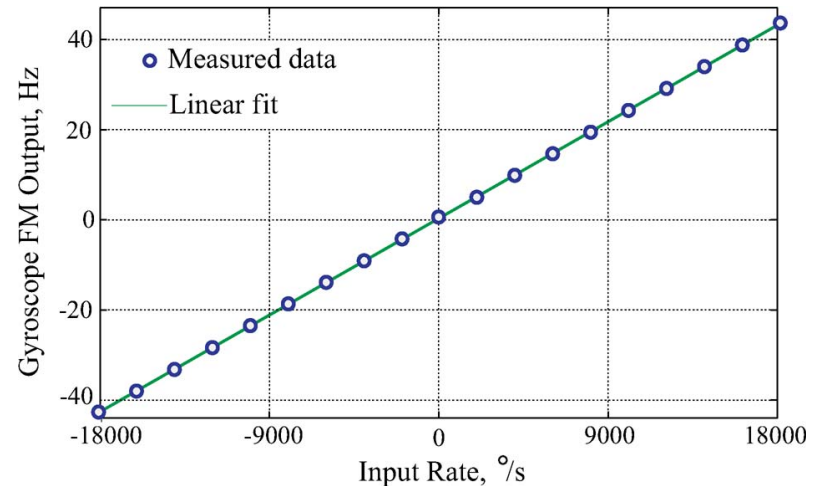

Fig. 15. Characterization of the FM-based rate sensor reveals less than $0.2 \%$ of nonlinearity in wide range of input range up to $18000 \mathrm{deg} / \mathrm{s}$.

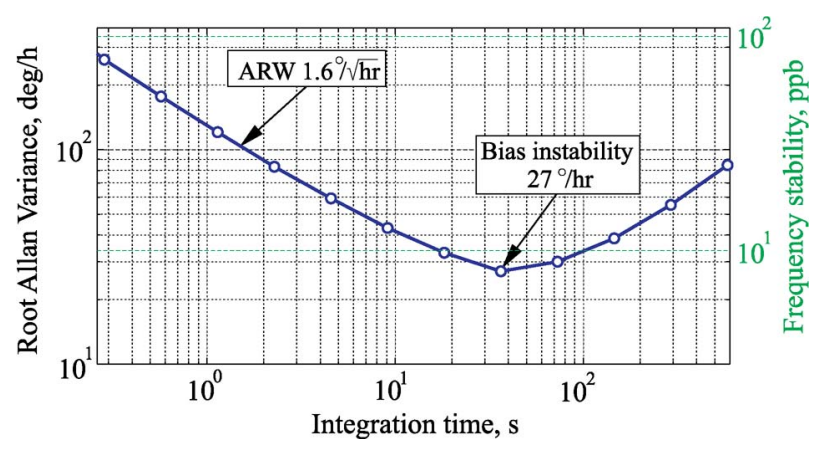

Fig. 16. Measured Allan variance of the FM sensor showing $1.6 \mathrm{deg} / \sqrt{\mathrm{h}}$ of ARW and 27-deg/h bias instability, for $18000-\mathrm{deg} / \mathrm{s}$ linear range.

factors [Fig. 14(b)]. Without any active temperature compensation, experimental characterization of the FM angular rate sensor at $25{ }^{\circ} \mathrm{C}$ and $70{ }^{\circ} \mathrm{C}$ revealed less than $0.2 \%$ response fluctuation (limited by the accuracy of the experimental setup) despite a $30 \%$ reduction of the $Q$ factor and a $5-\mathrm{Hz}$ drop of the nominal frequency caused by temperature dependence of Young's modulus.

Based on the theoretical analysis in Section II, the proposed FM gyroscope operation suggests a very wide sensor input range, limited only by the device natural frequency $\left(\Omega_{z}\right.$ needs to be less than $\omega$ ). In order to experimentally investigate this hypothesis, a vacuum-packaged QMG was mounted on an Ideal Aerosmith 1571 High-Speed Position and Rate Table System and characterized from 0 to $18000 \mathrm{deg} / \mathrm{s}$ (i.e., $50 \mathrm{r} / \mathrm{s}$ ). Without any compensation, the FM gyroscope demonstrated less than $0.2 \%$ nonlinearity (limited by noise, not systematic nonlinearity) throughout the entire range (Fig. 15).

Experimental characterization of the FM gyroscope reveals the following value of frequency split: $\lambda_{1}-\lambda_{2}=2 \kappa \Omega$, where $\kappa=0.864$. The coefficient $\kappa$ depends on the gyroscope design; for the QMG architecture shown in Fig. 6, this coefficient is calculated as $\kappa=M /\left(M+2 m_{\text {shuttle }}\right)$, where $M$ is the proof mass and $m_{\text {shuttle }}$ is the mass of the shuttle. For the particular physical layout, the theoretical value of $\kappa$ is equal to 0.870 . Noise performance of the FM sensor is limited by the frequency stability of the two modes of vibration in the gyroscope. Fig. 16 shows the measured Allan variance of the FM sensor showing angular random walk (ARW) of $1.6 \mathrm{deg} / \sqrt{\mathrm{h}}$, bias instability of
TABLE I

MEASUREd PARAMETERS OF THE QMG FOR AM, FM, AND AM/FM INTERCHANGEABLE OPERATION

\begin{tabular}{|l|c|}
\hline Parameter & Value \\
\hline Mechanical frequency & $2.177 \mathrm{kHz}$ (at $20{ }^{\circ} \mathrm{C}$, for $\Omega_{z}=0$ ) \\
\hline Q-factor maximum, at $-20{ }^{\circ} \mathrm{C}$ & 1.7 million \\
\hline Q-factor minimum, at $+20^{\circ} \mathrm{C}$ & $0.7 \mathrm{million}$ \\
\hline Q-factor minimum, at $+100^{\circ} \mathrm{C}$ & $1 \%$ \\
\hline$x / y$ axis $\Delta \mathrm{Q} / \mathrm{Q}$ & $2.367 \mathrm{mHz} /(\mathrm{deg} / \mathrm{s})$ \\
\hline Scale factor for FM mode & $<0.2 \%$ (noise limited) \\
\hline $\begin{array}{l}\text { Scale factor variation between } \\
\mathrm{T}=+20{ }^{\circ} \mathrm{C} \text { and } \mathrm{T}=+70^{\circ} \mathrm{C}\end{array}$ & $2.177 \mathrm{kHz}=7,800,000 \mathrm{deg} / \mathrm{s}$ \\
\hline Linear range FM mode, theory & $50 \mathrm{~Hz}=18,000 \mathrm{deg} / \mathrm{s}(\mathrm{setup} \mathrm{limited})$ \\
\hline Linear range FM mode, measured & $1.6 \mathrm{deg} / \sqrt{\mathrm{hr}} / 0.06 \mathrm{deg} / \sqrt{\mathrm{hr}}$ \\
\hline ARW FM/AM mode $[21]$ & $27 \mathrm{deg} / \mathrm{hr} /<1 \mathrm{deg} / \mathrm{hr}$ \\
\hline Bias instability FM/AM mode & $128 \mathrm{~dB}$ \\
\hline Dynamic range, FM mode & $156 \mathrm{~dB}$ \\
\hline $\begin{array}{l}\text { Dynamic range, } \\
\text { AM/FM interchangeable operation }\end{array}$ & \\
\hline
\end{tabular}

$27 \mathrm{deg} / \mathrm{h}$, and a dynamic range of $128 \mathrm{~dB}$ in the FM regime of operation (from $50 \mathrm{deg} / \mathrm{h}$ to $18000 \mathrm{deg} / \mathrm{s}$ ).

Previously, we reported two other regimes of operations for the QMG transducer. Whole-angle regime was demonstrated in [15], while interchangeable operation in whole-angle and AMrate modes was demonstrated in [11]. Detailed experimental characterization of QMG as conventional AM systems was studied in [21]. Rate characterization of QMG in conventional AM-rate regime demonstrated a bias instability of $<1 \mathrm{deg} / \mathrm{h}$ [21], providing a 156-dB dynamic range (from $<1 \mathrm{deg} / \mathrm{h}$ to $18000 \mathrm{deg} / \mathrm{s}$ ) of this transducer for the AM/FM interchangeable operation. Table I summarizes the parameters of QMG characterized in conventional AM and FM regimes as proposed in this paper.

\section{Conclusion}

We have proposed and demonstrated, for the first time, a quasi-digital angular rate sensor based on mechanical FM of angular rate input. This approach eliminates the gain-bandwidth tradeoff of conventional AM vibrating gyroscopes and may enable signal-to-noise ratio improvements through the use of high- $Q$-factor structures without limiting the measurement bandwidth and range. A differential frequency measurement enables simultaneous detection and decoupling of the input angular rate and the device temperature. In other words, the gyroscope becomes its own thermometer, eliminating thermal drift, lags, and hysteresis issues.

The novel approach was implemented and experimentally evaluated using a vacuum-packaged mode-matched SOI QMG rate sensor, with a $2.2-\mathrm{kHz}$ operational frequency and a measured $Q$ factor of one million. Advantages of the FMinstrumented QMG over the current state-of-the-art AMbased gyroscopes include an extremely wide linear range of $18000 \mathrm{deg} / \mathrm{s}$, dynamic ranges above $128 \mathrm{~dB}$, immunity to temperature variations, and robustness to external mechanical interferences and EMIs.

Recently, we have reported the experimental characterization of the QMG transducer as conventional analog AM system with a bias instability less than $0.9 \mathrm{deg} / \mathrm{h}$ [21]. The operation of the gyroscope in interchangeable mode (AM mode for low input range and FM mode for high input range) archives a dynamic range above $155 \mathrm{~dB}$. 


\section{ACKNOWLEDGMENT}

The authors would like to thank Dr. F. Heer of Zurich Instruments. The authors would also like to thank I. Prikhodko for the assistance with the experimental characterization of the prototype and I. Chepurko for the assistance with the interface electronics. Devices were designed and characterized at the MicroSystems Laboratory, University of California, Irvine.

\section{REFERENCES}

[1] N. Yazdi, F. Ayazi, and K. Najafi, "Micromachined inertial sensors," Proc. IEEE, vol. 86, no. 8, pp. 1640-1659, Aug. 1998.

[2] M. Weinberg, R. Candler, S. Chandorkar, J. Varsanik, T. Kenny, and A. Duwel, "Energy loss in MEMS resonators and the impact on inertial and RF devices," in Proc. TRANSDUCERS, 2009, pp. 688-695.

[3] M. F. Zaman, A. Sharma, Z. Hao, and F. Ayazi, "A mode-matched siliconyaw tuning-fork gyroscope with subdegree-per-hour Allan deviation bias instability," J. Microelectromech. Syst., vol. 17, no. 6, pp. 1526-1536, Dec. 2008.

[4] A. Sharma, M. F. Zaman, and F. Ayazi, "A 0.1 deg/hr bias drift micromechanical silicon gyroscope with automatic CMOS mode-matching," IEEE J. Solid-State Circuits, vol. 44, no. 5, pp. 1593-1608, May 2009.

[5] D. Xia, S. Chen, S. Wang, and H. Li, "Microgyroscope temperature effects and compensation-control methods," in Proc. IEEE Sensors, 2009, pp. 8349-8376.

[6] T. R. Albrecht, P. Grutter, D. Horne, and D. Rugar, "Frequency modulation detection using high-Q cantilevers for enhanced force microscope sensitivity," J. Appl. Phys., vol. 69, no. 2, pp. 668-673, Jan. 1991.

[7] A. A. Seshia, "Integrated micromechanical resonant sensors for inertial measurement systems," Ph.D. dissertation, Univ. California, Berkeley, CA, 2002.

[8] A. A. Seshia, R. Howe, and S. Montague, "An integrated microelectromechanical resonant-output gyroscope," in Proc. IEEE MEMS, Las Vegas, NV, 2002, pp. 722-726.

[9] C. Comi, A. Corigliano, G. Langfelder, A. Longoni, A. Tocchio, and B. Simoni, "A high sensitivity uniaxial resonant accelerometer," in Proc. IEEE MEMS, 2010, pp. 260-263.

[10] S. A. Zotov, I. P. Prikhodko, A. A. Trusov, and A. M. Shkel, "Frequency modulation based angular rate system," in Proc. IEEE MEMS, Cancun, Mexico, Jan. 23-27, 2011, pp. 557-580.

[11] A. A. Trusov, I. P. Prikhodko, S. A. Zotov, A. R. Schofield, and A. M. Shkel, "Ultra-high Q silicon gyroscopes with interchangeable rate and whole angle modes of operation," in Proc. IEEE Sensors, 2010, pp. 864-867.

[12] A. A. Trusov, S. A. Zotov, and A. M. Shkel, "High range digital angular rate sensor based on frequency modulation," U.S. Patent pending, UC Case No. 2011-199.

[13] D. Lynch, "Coriolis vibratory gyroscope," in Proc. Symp. Gyro Technol., Stuttgart, Germany, 1998, pp. 1.0-1.14.

[14] A. M. Shkel, C. Acar, and C. Painter, "Two types of micromachined vibratory gyroscopes," in Proc. IEEE Sensors, 2005, pp. 531-536.

[15] I. P. Prikhodko, S. A. Zotov, A. A. Trusov, and A. M. Shkel, "Foucault pendulum on a chip: Angle measuring silicon MEMS gyroscope," in Proc. IEEE MEMS Conf., Cancun, Mexico, 2011, pp. 161-164.

[16] J. R. Vig, Quartz Crystal Resonators and Oscillators; For Frequency Control and Timing Applications-A Tutorial, Jan. 2007, Rev. 8.5.3.6.

[17] A. R. Schofield, A. A. Trusov, and A. M. Shkel, "Versatile sub-mTorr vacuum packaging for the experimental study of resonant MEMS," in Proc. IEEE MEMS, 2010, pp. 516-519.

[18] B. Kim, M. Hopcroft, R. Candler, C. Jha, M. Agarwal, R. Melamud, S. Chandorkar, G. Yama, and T. Kenny, "Temperature dependence of quality factor in MEMS resonators," J. Microelectromech. Syst., vol. 17, no. 3, pp. 755-766, Jun. 2008.

[19] A. A. Trusov, S. A. Zotov, and A. M. Shkel, "Electrostatic regulation of quality factor in tuning fork MEMS," in Proc. IEEE Sensors, 2011, pp. 20-23.
[20] A. A. Trusov and A. M. Shkel, "Capacitive detection in resonant MEMS with arbitrary amplitude of motion," J. Micromech. Microeng., vol. 17, no. 8, pp. 1583-1592, Jul. 2007.

[21] I. P. Prikhodko, S. A. Zotov, A. A. Trusov, and A. M. Shkel, "Sub degree per hour silicon MEMS rate sensor with 1 million Q-factor," in Proc. TRANSDUCERS, 2011, pp. 2809-2812.

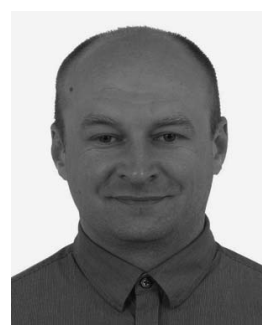

Sergei A. Zotov (M'11) received the M.S. and Ph.D. degrees in mechanical engineering and control systems from Tula State University, Tula, Russia, in 1999 and 2002, respectively.

He is currently a Postdoctoral Scientist with the MicroSystems Laboratory, University of California, Irvine, where he is responsible for the design, fabrication, and testing of micromachined devices and systems for inertial navigation. Over the last ten years, his focus has been on MEMS accelerometers and gyroscopes. He is the holder of eight Russian patents and three U.S. patents pending.

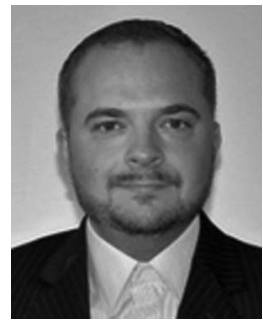

Alexander A. Trusov (S'05-M'09) received the B.S. and M.S. degrees in applied mathematics and mechanics from Moscow State University, Moscow, Russia, in 2004, and the M.S. and Ph.D. degrees in mechanical and aerospace engineering from the University of California, Irvine, in 2006 and 2009, respectively.

$\mathrm{He}$ is currently a Project Scientist with the Department of Mechanical and Aerospace Engineering, University of California, Irvine, where he is the Principal Investigator (PI) and Co-PI of five Department of Defense-sponsored research programs. He has published 13 journal and over 30 conference papers on MEMS and signal processing and has seven pending U.S. patents on MEMS design, fabrication, and control. His research interests include design, modeling, fabrication, and vacuum packaging of micromachined inertial systems, design of characterization experiments, and statistical data processing and analysis.

Dr. Trusov is a member of the American Society of Mechanical Engineers. He serves as a Reviewer for major journals in the fields of MEMS and sensors and is a member of the MEMS and Associated Microsystems Organizing Committee of the International Microelectronics and Packaging Society Device Packaging 2011 and 2012 conferences. He was a recipient of the First-Place Student Design Contest Award at the 2011 International System-on-Chip Conference and the Outstanding Paper Award at Transducers 2011 for his work on low-dissipation MEMS gyroscopes.

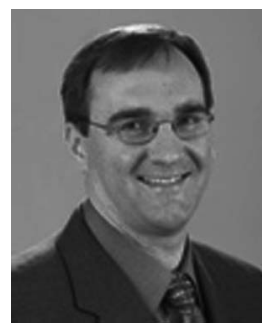

Andrei M. Shkel (S'95-A'98-M'07-SM'08) received the Diploma degree (with excellence) in mechanics and mathematics from Moscow State University, Moscow, Russia, in 1991, and the Ph.D. degree in mechanical engineering from the University of Wisconsin, Madison, in 1997.

$\mathrm{He}$ is currently a Program Manager with the Microsystems Technology Office, Defense Advanced Research Projects Agency, Arlington, VA; he is serving in this capacity while on leave from his faculty position as a Professor in the Department of Mechanical and Aerospace Engineering, University of California, Irvine (UCI), where he is also the Director of the MicroSystems Laboratory. He is the holder of 15 U.S. and international patents. His professional interests are reflected in more than 120 publications.

Dr. Shkel is an Editor of the Journal of Microelectromechanical SYSTEMS. He was the recipient of the 2009 Research Award from the IEEE Sensors Council, the 2006 UCI Research Award, a 2005 National Science Foundation CAREER Award, and the 2002 George E. Brown, Jr., Award. 